\title{
Medical Oncology
}

\section{Phase II Trial of Carboplatin, Everolimus, and Prednisone in Metastatic \\ Castration-resistant Prostate Cancer Pretreated With Docetaxel Chemotherapy: A Prostate Cancer Clinical Trial Consortium Study}

\author{
Ulka Vaishampayan, Daniel Shevrin, Mark Stein, Lance Heilbrun, Susan Land, Karri Stark, \\ Jing Li, Brenda Dickow, Elisabeth Heath, Daryn Smith, and Joseph Fontana
}

OBJECTIVE

To conduct a phase II trial of the combination of carboplatin, prednisone, and everolimus in metastatic castrate-resistant prostate cancer (mCRPC) as mTOR inhibition can overcome resistance to chemotherapy in prostate cancer.

METHODS

Patients with progressive mCRPC pretreated with docetaxel-based regimen were eligible. Performance status of $0-1$ and adequate bone marrow, renal, and liver function were required. Primary end point was time to progression. Treatment consisted of carboplatin (starting dose equal to area under the curve (AUC of 5) intravenously every 21 days along with oral everolimus $5 \mathrm{mg}$ once daily and prednisone $5 \mathrm{mg}$ twice daily.

RESULTS Twenty-six patients were enrolled with median age of 69 years with 8 patients of African American origin. Grade 3 or 4 thrombocytopenia or neutropenia in 4 of 6 initial patients required dose adjustment of carboplatin to AUC of 4 for subsequent patients. There were no pharmacokinetic interactions between carboplatin and everolimus. The median time to progression was 2.5 months (90\% confidence interval [CI], 1.8-4.3 months), and median overall survival was 12.5 months (90\% CI, 7.7-18.7 months). Of 10 patients, 8 that demonstrated positive nuclear phosphorylated AKT (pAKT) staining on immunohistochemistry progressed within 9 weeks, whereas 2 patients with negative staining continued without progression for prolonged durations of 30 and 48 weeks. TSC1 gene mutations did not correlate with clinical outcome.

CONCLUSION The addition of the mTOR inhibitor everolimus to carboplatin demonstrated minimal clinical efficacy in metastatic prostate cancer. pAKT testing warrants further evaluation as a predictive marker of response to everolimus therapy. UROLOGY n: - - -, 2015 . (c) 2015 Elsevier Inc.

$\mathrm{R}$ ecently, novel agents such as cabazitaxel, enzalutamide, radium-223, and abiraterone plus prednisone have each demonstrated overall survival (OS) improvement in post-docetaxel mCRPC. ${ }^{1-4}$

Clinicaltrials.gov registration: NCT01051570.

Financial Disclosure: Ulka Vaishampayan received research support, consulting, and honoraria from Novartis Inc, and Brenda Dickow is a paid consultant to Novartis Inc. The remaining authors declare that they have no relevant financial interests.

Funding Support: This study was supported in part by grants from Department of Defense W81XWH-09-1-0143, NIH Cancer Center Support Grant CA-22453, and Novartis Inc.

From the Department of Oncology, Department of Medicine, Barbara Ann Karmanos Cancer Institute, Wayne State University, Detroit, MI; the Northshore University Health System, Evanston, IL; the Cancer Institute of New Jersey, New Brunswick, NJ; the Biostatistics Core, Barbara Ann Karmanos Cancer Institute, Detroit, MI; and the John D. Dingell Veterans Medical Center, Detroit, MI

Address correspondence to: Ulka Vaishampayan, M.D., 4 Hudson Webber Cancer Research Center, 4100 John R, Detroit, MI 48201. E-mail: vaishamu@karmanos.org

Submitted: June 5, 2015, accepted (with revisions): August 13, 2015
Despite the availability of all these agents, few long-term remissions are induced and mCRPC remains an incurable, terminal malignancy. Resistance to therapy and emergence of neuroendocrine variants are frequent reasons for therapeutic failure in mCRPC. Platinumbased chemotherapy has been utilized for the treatment of resistant mCRPC, or for therapy of small-cell carcinoma of the prostate. In docetaxel refractory setting, the addition of carboplatin to docetaxel demonstrated a response rate in the range of $18 \%-20 \%$ and median duration of response of 5.7 months. ${ }^{5}$ Other platinum agents such as satraplatin and oxaliplatin have also shown efficacy in docetaxel refractory mCRPC. ${ }^{6}$ The use of platinum-based therapy is currently confined to refractory or neuroendocrine presentations of the disease. 
The Prostate Cancer Clinical Trials Consortium conducted a phase II trial of docetaxel and carboplatin which revealed a prostate-specific antigen (PSA) response rate ( $\geq 50 \%$ decline) of $18 \%$, median progression-free survival (PFS) of 3 months, and median OS of 12 months. ${ }^{7}$ This study established the modest efficacy of carboplatin therapy in disease progression after docetaxel. ${ }^{7}$ The addition of everolimus to cisplatin demonstrated enhanced sensitivity and increased antiproliferative and apoptotic effects. ${ }^{8}$ The combination was evaluated in a clinical setting and was well tolerated with no overlapping toxicities. ${ }^{8}$ Clinical responses were observed in multiple malignancies such as carcinoid and oropharyngeal squamous cell carcinomas. ${ }^{8}$

Everolimus (RAD001, Novartis Inc) is an mTOR pathway inhibitor that has proven efficacy and received Food and Drug Administration approval in metastatic renal, breast, and pancreatic neuroendocrine cancers. ${ }^{9}$ Loss of PTEN expression has also been reported to be associated with an increase in PI3 kinase activity and mTOR activity. ${ }^{10}$ mTOR inhibition in PTEN-negative prostate tumors results in decreased S6 activity and antiproliferative effects.

mTOR inhibition was very effective as a single agent in both PTEN-positive (DU-145) and mutant/PTENnegative (PC-3) prostate cancer cell lines, and xenograft models, and demonstrated superior efficacy than single-agent mitoxantrone or docetaxel therapy. ${ }^{11}$ The combination of mitoxantrone with mTOR inhibitor, administered sequentially, showed increased and sustained antitumor effect, as compared to the use of mitoxantrone alone in PC-3 prostate cancer cell lines and xenografts. ${ }^{12}$ In addition to the antiproliferative effect, inhibition of mTOR also demonstrated inhibition of repopulation of tumor cells. Clinically, this is likely to translate to sustained responses and durable remission. In fact, mTOR inhibition in prostate cancer cells demonstrated reinduction of sensitivity to chemotherapy even where resistance was noted related to PTEN-negative PC-3 cell lines and xenografts. ${ }^{10,12,13}$

The PI3 kinase pathway activity is reported to result in resistance to therapies that block the androgen receptor signaling. Hence, as castrate-resistant state evolves, the PI3 kinase and downstream mTOR pathways are likely to be some of the key drivers of disease progression and resistance development. The rationale of everolimus having the potential of overcoming resistance to chemotherapy, synergy with platinum, ${ }^{13}$ and inhibiting repopulation of prostate cancer cells, led to a phase II trial of everolimus with carboplatin in mCRPC.

\section{METHODS}

\section{Patient Eligibility}

Eligibility included histologically confirmed prostate adenocarcinoma with measurable or evaluable, or radiologically evident metastases. Objective evidence of progression was required, based on increase in dimensions of measurable disease, or new bone lesions, or rising PSA despite androgen deprivation therapy and antiandrogen withdrawal when applicable. Patients with rising PSA only had to demonstrate at least 2 successive elevations at a minimum interval of 1 week. A minimum PSA of $5 \mathrm{ng} / \mathrm{mL}$ or new areas of bony metastases on bone scan were required for patients with no measurable disease. Prior docetaxelbased chemotherapy was a prerequisite, but not more than 2 prior chemotherapy regimens (unless given in combination with docetaxel) for metastatic disease were allowed. All patients had to have documented castrate levels of testosterone at $50 \mathrm{ng} / \mathrm{mL}$ or less. Patients on antiandrogens had to be off for a minimum of 4 weeks for flutamide, and 6 weeks for bicalutamide or nilutamide. Prior radiation therapy had to be completed at least 28 days before enrollment. Eastern Cooperative Oncology group performance status of 0-2, life expectancy of at least 12 weeks, and normal renal, liver, and bone marrow function were required. Patients with fasting serum cholesterol $\geq 300 \mathrm{mg} / \mathrm{dL}$ or $\geq 7.75 \mathrm{mmol} / \mathrm{L}$ and fasting triglycerides $\geq 2.5 \times$ upper limit of normal were excluded. All patients were required to provide signed written informed consent, and regulatory approval was maintained. The clinical trial was registered at www.ct.gov [NCT01051570].

\section{Treatment Plan}

Carboplatin was infused on an outpatient basis at a dose of area under the curve (AUC) of 5 as per the Calvert's formula, intravenously over 60 minutes every 21 days. Everolimus was administered orally at a dose of $10 \mathrm{mg}$ daily, and prednisone $5 \mathrm{mg}$ was given orally twice daily. If $\geq 3$ of the first 6 patients enrolled required carboplatin dose reduction after cycle 1 because of grade 3 or 4 cytopenias, then all subsequent patients would be started with an AUC of 4 dose of carboplatin.

\section{Study Design}

The study design was a 1-stage, fixed sample size phase II design, with its primary statistical objective to evaluate the time to progression (TTP). Secondary objectives were to evaluate the safety of the combination, to assess the PSA response rates, and to evaluate the OS outcome.

The objectives for correlative testing consisted of investigation of the association of TTP and PSA response rate with phospho mTOR, phosphorylated AKT (pAKT), and p70S6 in archival tumor tissue. The association of circulating tumor cell (CTC) count with time to event end points was explored. The pharmacokinetics of the combination were evaluated, and TSC1 gene mutations were tested in the patients who had archival tumor tissue available.

All patients registered on the protocol and completing a minimum of 1 cycle of therapy followed by clinical, radiologic, or PSA assessment of disease status were considered response evaluable. Patients underwent imaging studies for tumor assessment at baseline and every 2 cycles thereafter. PSA level was determined on day 1 of each cycle. RECIST $1.1^{14}$ criteria were used to categorize response and progression in measurable disease, and Prostate Cancer Working Group criteria II were used to determine progression in patients with bone-only metastases. ${ }^{15}$ Symptomatic skeletal event was considered disease progression and was defined as occurrence of new pathologic bone fractures (vertebral or nonvertebral), spinal cord compression, requirement of surgery or radiation therapy to bone metastases (including the use of radioisotopes), or a requirement to change antineoplastic therapy to treat bone pain or other related symptoms. 
Toxicity was monitored every 3 weeks, except in cycle 1 when weekly complete blood counts were conducted. All patients registered on the protocol and starting therapy with the protocol medication were considered toxicity evaluable.

\section{Statistical Methods}

Statistical Design. A 1-sample survival-type design was used based on the methods of Lawless. ${ }^{16}$ Assuming exponentially distributed TTP, the relevant test utilizes an approximate $\mathrm{Z}$ statistic. A cube root transformation of the hazard provides good small sample properties. The median TTP achievable with the regimen was hypothesized to exceed an assumed population reference (ie, control) value of 2.5 months. A clinically meaningful improvement in median TTP would be 1.5 months over that, that is, if carboplatin and everolimus had a true median TTP of 4.0 months (ie, a $60 \%$ improvement). With power set at 0.90 and a relaxed alpha of 0.15 (1-sided), the required sample size was 26 patients.

Analysis. Confidence interval (CI) estimates of PSA response rate and grade 3-4 toxicity rates were calculated using Wilson's method. Owing to the small sample sizes, TTP and OS statistics (eg, median, 6-month rate) were estimated more conservatively using linear interpolation among successive event times on the Kaplan-Meier curves. ${ }^{17}$ Censored TTP or censored OS curves were compared by level of CTC ( $<5$ vs $\geq 5$ per $7.5 \mathrm{~mL}$ of blood) using the log-rank test. The Cox proportional hazards model was used to estimate the hazard ratio for progression (and separately for death) for patients with $\geq 5$ CTC compared to patients with $<5$ CTC. The proportional hazards assumption was examined for the dichotomous CTC predictor variable by inspection of $\log (-\log )$ (survival time) plots, and by inspection of smoothed hazard functions estimated with the Epanechnikov kernel smoother. All statistical analyses were performed and statistical graphics generated using SAS 9.3 software.

\section{Correlative Tests}

Pharmacokinetics. Detailed methodology is included in supplemental information (Supplementary Table 2S).

Biomarker assessment. Immunohistochemistry (IHC) staining for nuclear and cytoplasmic AKT, pAKT, mTOR, and p70S6 was performed on archival tumor tissue by citrate buffer antigen retrieval protocol. CTC counts by Veridex technique were done before therapy and 1 week after therapy.

Genomic testing on TSC gene. We tested tuberous sclerosis gene 1 (TSC1) mutations in tumor tissue of the patients enrolled in this study and examined the association of TSC1 mutations with clinical outcome.

DNA Isolation. DNA was isolated from archival samples using the Qiagen EZ1 Advanced using magnetic bead technology, with the "purification of DNA from paraffin-embedded tissue" protocol, the EZ1 DNA Tissue Kit, and the EZ1 DNA Paraffin card. The purified DNA was quantified by the Quantifiler by Life Technologies and purity based on the inhibition of polymerase chain reaction.

DNA Sequencing. The TSC1 exons were amplified. ${ }^{18}$ Their updated primer sequences were utilized (private communication). DNA sequencing reactions were carried out using the 0.5X protocol for ABI PRISM BigDye Terminator Cycle
Sequencing Ready Reaction Kit (Applied Biosystems). The sequencing extension products will be purified utilizing Sephadex. The purified products were analyzed on an ABI PRISM 3730 DNA Analyzer using a 50-cm capillary array.

\section{RESULTS}

Twenty-six patients were enrolled and evaluable on the trial. Patient characteristics and toxicities are summarized in Supplementary Tables $1 S$ and 2S. Predominant toxicities noted were grade 3 or 4 thrombocytopenia, and neutropenia in 4 of 6 initial patients, requiring dose adjustment of carboplatin to AUC of 4 in subsequent cycles for enrolled patients and as starting dose for new patients. Nineteen patients had measurable disease. No objective responses were noted, but 8 patients demonstrated stable disease. PSA decline of $30 \%$ or greater was noted in 4 patients of which 1 patient had a PSA decline of $>90 \%$. A median of 3 cycles (range, 1-16 cycles) was administered. The carboplatin PK levels and AUC readings were not affected by everolimus. Median AUC for carboplatin were 5.9 (range, 4.3-11.0) and 4.5 (range, 4.1-7.1) $\mathrm{mg} / \mathrm{mL} / \mathrm{minute}$ when carboplatin was given alone and in combination with everolimus, respectively (Supplementary Table 3S). Everolimus did not impact the PK of carboplatin. The median TTP was 2.5 months (90\% CI, 1.8-4.3 months; Table 1 and Supplementary Fig. 1S), and the median OS was 12.5 months (90\% CI, 7.7-18.7 months; Table 1). The study did not meet the primary end point of true TTP of 4 months as specified in the statistical plan.

\section{Biomarker Results}

Circulating Tumor Cell Counts. Median baseline CTC $(\mathrm{n}=18)$ was 30 (range, $0-2372)$. Of 18 patients, 5 had favorable CTC (CTC $<5 / 7.5 \mathrm{~mL}$ ) pretherapy. Patients with treatment duration of 6 or more cycles demonstrated a larger magnitude of reduction in post-therapy CTC after cycle 1 as compared to baseline, with a median decrease of $63 \%$ (range, $11 \%-100 \%$; Fig. 1).

Pharmacodynamic Testing. IHC staining for AKT, pAKT, mTOR, and p70S6 was performed on 10 patients with availability of archival tumor tissue (Fig. 2). Negative IHC staining for $\mathrm{pAKT}$ was noted on 2 patients who continued on therapy for durations of 30 and 48 weeks, and positive staining was noted in the remaining 8 patients who were on therapy for 9 weeks or less.

TSC1 Gene Mutation Testing. TSC1 gene mutation testing was conducted on 7 archival tumor tissue samples. Four patients demonstrated exon 10 mutations of which 2 patients had a TTP of 8 and 17 months (Table 2). The other 2 patients with exon 10 mutations revealed a TTP of 1.5 months each.

\section{COMMENT}

Platinum-based therapy has been used in refractory $\mathrm{mCRPC}$ and in the neuroendocrine/small-cell variants of 
Table 1. Response rates and time to event end points

\begin{tabular}{|c|c|c|c|c|c|}
\hline End point & $\mathrm{N}$ & Events & Point Estimate & \multicolumn{2}{|c|}{ 90\% Confidence Interval } \\
\hline \multicolumn{6}{|l|}{ PSA decline } \\
\hline$>30 \%$ Decrease & 26 & 4 & $15 \%$ & 7 & 30 \\
\hline Measurable disease response & 19 & & & & \\
\hline $\mathrm{CR} / \mathrm{PR}$ & 19 & 0 & 0 & 0 & 0 \\
\hline Stable disease & 19 & 8 & $42 \%$ & 26 & 61 \\
\hline Time to progression & 26 & 25 & & & \\
\hline Median & & & $2.5 \mathrm{mo}$ & $1.8 \mathrm{mo}$ & $4.3 \mathrm{mo}$ \\
\hline 6-mo rate & & & $30 \%$ & 14 & 45 \\
\hline 12-mo rate & & & $8 \%$ & 0 & 18 \\
\hline Cycle 1 , day 1 CTC $<5$ & 5 & 5 & & & \\
\hline Median & & & $2.9 \mathrm{mo}$ & $1.0 \mathrm{mo}$ & $18.3 \mathrm{mo}$ \\
\hline 6-mo rate & & & $33 \%$ & 0 & 69 \\
\hline 12-mo rate & & & $17 \%$ & 0 & 47 \\
\hline Cycle 1 , day 1 CTC $\geq 5$ & 13 & 12 & & & \\
\hline Median & & & $2.2 \mathrm{mo}$ & $1.5 \mathrm{mo}$ & $11.6 \mathrm{mo}$ \\
\hline 6-mo rate & & & $27 \%$ & 5 & 48 \\
\hline 12-month rate & & & $9 \%$ & 0 & 24 \\
\hline Overall survival & 26 & 25 & & & \\
\hline Median & & & $12.5 \mathrm{mo}$ & $7.7 \mathrm{mo}$ & $18.7 \mathrm{mo}$ \\
\hline 6-mo rate & & & $77 \%$ & 64 & 91 \\
\hline 12-morate & & & $54 \%$ & 38 & 70 \\
\hline Cycle 1 , day 1 CTC $<5$ & 5 & 4 & & & \\
\hline Median & & & $11.3 \mathrm{mo}$ & $3.9 \mathrm{mo}$ & —* \\
\hline 6-mo rate & & & $76 \%$ & 39 & 100 \\
\hline 12-mo rate & & & $42 \%$ & 6 & 78 \\
\hline Cycle 1 , day 1 CTC $\geq 5$ & 13 & 13 & & & \\
\hline Median & & & $8.8 \mathrm{mo}$ & $4.0 \mathrm{mo}$ & $17.3 \mathrm{mo}$ \\
\hline 6-mo rate & & & $62 \%$ & 40 & 84 \\
\hline 12-mo rate & & & $42 \%$ & 19 & 65 \\
\hline
\end{tabular}

$\mathrm{CR}$, complete response; CTC, circulating tumor cell; PR, partial response; PSA, prostate-specific antigen.

* Cannot be calculated because of the small sample size and the censoring pattern.

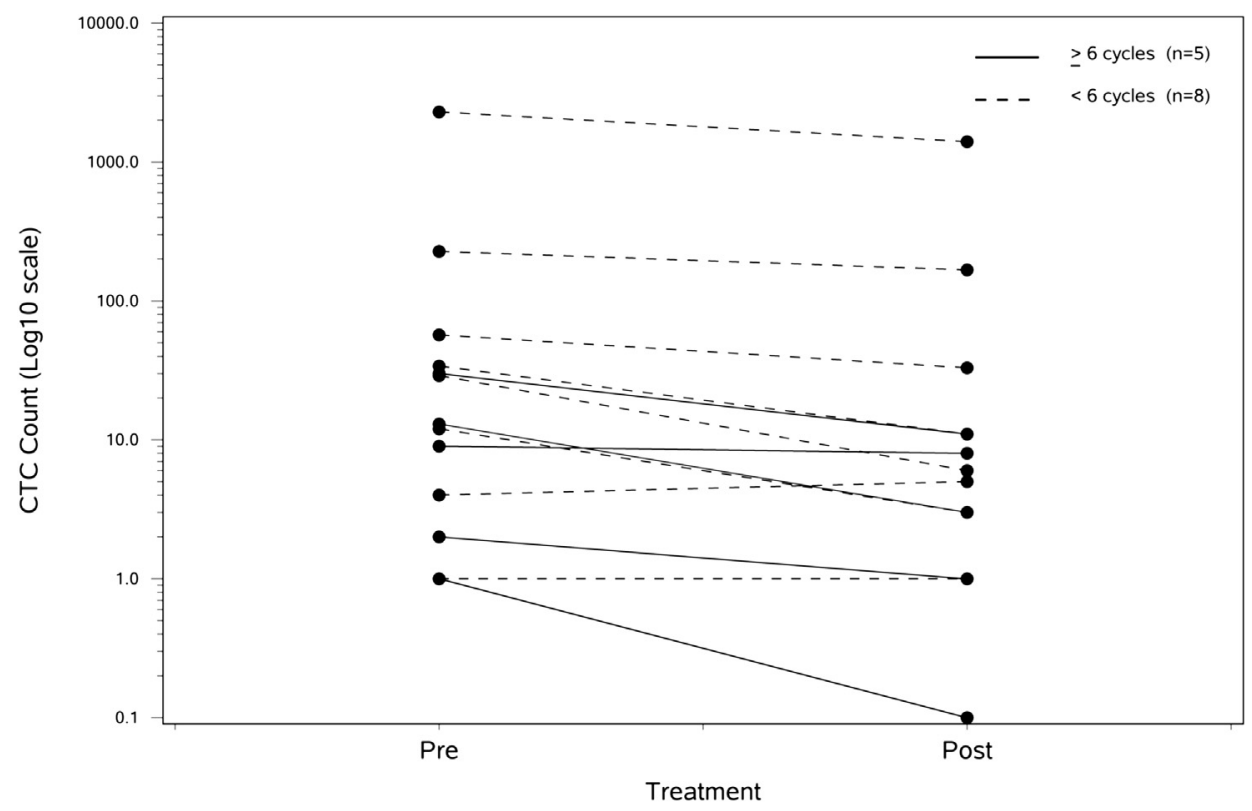

Figure 1. Circulating tumor cell measurement pretherapy and post-therapy.

prostate cancer. Carboplatin therapy has demonstrated efficacy in docetaxel refractory patients. The combination of carboplatin and mTOR inhibitor everolimus has been evaluated in multiple malignancies. In malignant melanoma, a combination of carboplatin, paclitaxel, and everolimus was evaluated in a phase II trial ${ }^{19}$ with $17 \%$ response and a disappointing median PFS of 4 months. In xenograft models, synergy was observed between chemotherapy and everolimus in breast and endometrial cancer. However, clinically the combinations have 


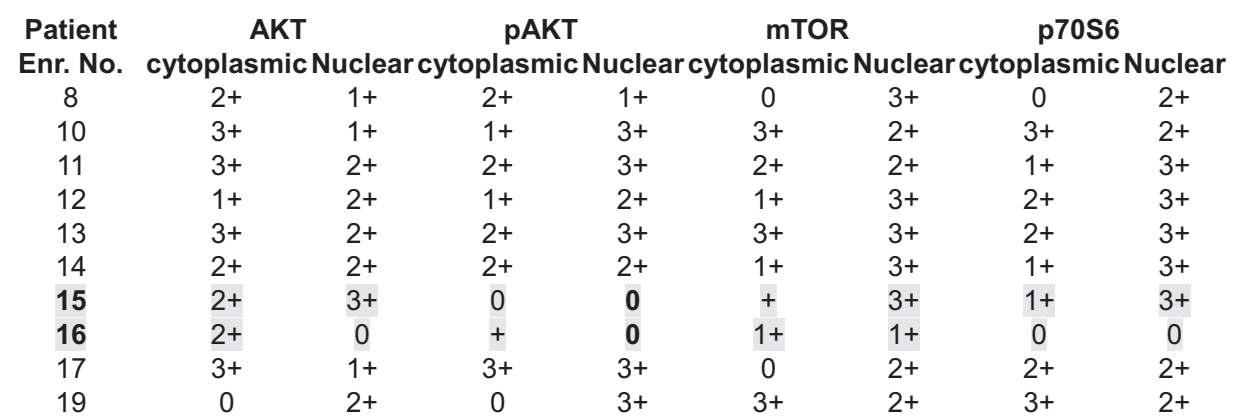

Figure 2. Immunohistochemical staining of cytoplasmic and nuclear pAKT, p70S6, and phospho mTOR. Patients 15 and 16 (highlighted) were on therapy for 30 and 48 weeks, respectively, whereas other patients were on therapy for $\leq 9$ weeks.

Table 2. TSC1 gene mutations on archival tumor tissue in 7 patient samples and TTP and OS

\begin{tabular}{lccc}
\hline $\begin{array}{l}\text { Sample } \\
\text { Number }\end{array}$ & Mutations & TTP & OS \\
8 & Exon 1, 10, 19, and 21 & $1.5 \mathrm{mo}$ & $7 \mathrm{mo}$ \\
11 & Exon 1, 6, 7, 10, 12, & $1.5 \mathrm{mo}$ & $12.1 \mathrm{mo}$ \\
& 15,19, and 22 & & \\
12 & Exon 5, 10, 14, and 19 & $2.5 \mathrm{mo}$ & $4.8 \mathrm{mo}$ \\
14 & $\quad$ Exon 15 & $1.5 \mathrm{mo}$ & $25.5 \mathrm{mo}$ \\
15 & Exon 3, 5, 6, 10, 11, & $8 \mathrm{mo}$ & $19.6 \mathrm{mo}$ \\
& 19,21, and 23 & & \\
16 & Exon 1 and 10 & $17 \mathrm{mo}$ & $36.9 \mathrm{mo}$ \\
17 & No variations & $1.5 \mathrm{mo}$ & $4.5 \mathrm{mo}$ \\
\hline
\end{tabular}

OS, overall survival; TTP, time to progression.

yielded suboptimal results. In carboplatin- and etoposidetreated patients post-docetaxel, the median PFS was reported to be about 3 months as reported in a trial evaluating 75 patients with mCRPC, and features of variant disease. ${ }^{20}$ Our study showed a comparable PFS and OS as reported in the variant mCRPC trial noted above. In addition, it should be recognized that the patient population enrolled in this study had multiple poor prognostic features such as $69 \%$ of the patients with Gleason score 8 or higher, $62 \%$ with bone pain, $38 \%$ progressing after 2 prior chemotherapy regimens, and $31 \%$ with visceral metastases. The main toxicity noted was myelosuppression, and similar to another study assessing the combination of carboplatin and everolimus, dose reduction to AUC of 4 of carboplatin was required. However, everolimus did not have a significant impact on carboplatin levels, as shown by the PK testing conducted in our trial. The pharmacodynamic biomarkers tested were exploratory, but maybe hypotheses-generating for future studies of mTOR inhibition in mCRPC.

The lack of patient selection may have a critical role to play in accounting for the lack of clinical efficacy seen with everolimus therapy. A phase II study of everolimus in advanced urothelial cancer was closed because of lack of activity after accrual of 14 patients. ${ }^{21}$ The tumor genome of 1 patient who had a durable remission for over 2 years was studied and revealed TSC1 gene mutations. Patients with TSC1 mutant tumors remained in the study longer (median, 7.7 months vs 2.0 months) than those without the mutations. ${ }^{21}$ The results suggest that TSC mutation-enriched population is more likely to benefit from mTOR-directed therapies. We conducted TSC1 mutation testing and noted that 1 patient with prolonged remission (17 months) had exon 1 and 10 mutations. A Swiss trial of 37 patients with mCRPC who were treated with everolimus revealed that 13 of 37 patients were progression free at 12 weeks with PSA decline $>30 \%$ noted in 6 patients. PTEN deletion was associated with clinical response and a longer PFS. ${ }^{22}$ In our trial, pharmacodynamic testing revealed that undetectable pAKT on IHC of tumor tissue, seen in 2 of 10 patients, was associated with longer PFS. A combination of bicalutamide and everolimus was evaluated in a clinical trial in mCRPC. Only 2 of the 36 patients enrolled demonstrated a PSA response, and the median PFS was 8.7 weeks. ${ }^{23}$ The discrepancy noted between preclinical and clinical results can probably be avoided in future trials of $\mathrm{mTOR}$ inhibitors, by better patient selection on the basis of some or all of the above biomarkers detected such as PTEN deletion, TSC1 mutations, and lack of pAKT IHC staining.

In prostate cancer, the roles of the androgen receptor (AR) and mTOR are closely interlinked in promoting tumor proliferation. Dihydrotestosterone stimulation of LNCaP cells revealed increased cyclin D1 protein expression and cell cycle progression via activation of mTOR. ${ }^{24}$ The PI3 kinase pathway is also responsible for activation of AR, especially noted in the prostate cancers with PTEN loss. Transcriptional activity of AR was increased when the PI3 kinase pathway was inhibited at different levels; however, antitumor effect was observed. In the androgen-responsive prostate cancer cell line LNCaP, both testosterone and the mTOR inhibitor rapamycin synergistically activated androgen target genes. Despite increased androgen signaling, rapamycin treatment reduced LNCaP cell growth. The rapamycin derivative CCI-779 was reported to decrease the growth of CWR22 prostate cancer xenografts despite increasing AR target gene expression. Hence, the mTOR/PI3 kinase pathway effect preclinically superseded the proliferative effects of androgen receptor activation. ${ }^{25}$

These findings suggest that inhibition of the PI3 kinase pathway activates AR signaling. Despite the increase in 
AR signaling which has proliferative effects, the result of PI3 kinase pathway inhibition is antiproliferative in prostate cancer. These findings suggest that the PI3 kinase pathway is dominant over AR signaling in castrateresistant disease. A dual inhibitor of PI3 kinase and mTOR revealed superior results over that of single-agent activity of each of these agents. ${ }^{26}$ Another resistance pathway in prostate cancer was characterized by the emergence of CSCs with AKT upregulation and elevated hypoxia-inducible factor1-alpha levels and hypoxiainducible factor target gene expression. These resistance mechanisms can be postulated as possible reasons why the patients with lack of pAKT expression on their archival tumor tissue had the longest benefit from mTOR inhibition in our study.

\section{CONCLUSION}

The combination of carboplatin and everolimus demonstrated minimal efficacy in metastatic CRPC. Further evaluation of this regimen in prostate cancer is not recommended unless a specific biomarker can be isolated to help guide selection of therapy. The absence of pAKT may be a biomarker predictive of response and clinical benefit. Future investigations developing effective tools to individualize therapy based on clinical and molecular markers are likely to optimize outcome.

\section{References}

1. de Bono JS, Oudard S, Ozguroglu M, et al. Prednisone plus cabazitaxel or mitoxantrone for metastatic castration-resistant prostate cancer progressing after docetaxel treatment: a randomised openlabel trial. Lancet. 2010;376:1147-1154.

2. de Bono JS, Logothetis CJ, Molina A, et al. Abiraterone and increased survival in metastatic prostate cancer. N Engl J Med. 2011; 364:1995-2005.

3. Scher HI, Fizazi K, Saad F, et al. Increased survival with enzalutamide in prostate cancer after chemotherapy. N Engl J Med. 2012; 367:1187-1197.

4. Parker C, Nilsson S, Heinrich D, et al. Alpha emitter radium-223 and survival in metastatic prostate cancer. N Engl J Med. 2013; 369:213-223.

5. Nakabayashi M, Sartor O, Jacobus S, et al. Response to docetaxel/ carboplatin-based chemotherapy as first- and second-line therapy in patients with metastatic hormone-refractory prostate cancer. BJU Int. 2008;101:308-312.

6. Bhargava A, Vaishampayan UN. Satraplatin: leading the new generation of oral platinum agents. Expert Opin Investig Drugs. 2009; 18:1787-1797.

7. Ross RW, Beer TM, Jacobus S, et al. A phase 2 study of carboplatin plus docetaxel in men with metastatic hormone-refractory prostate cancer who are refractory to docetaxel. Cancer. 2008;112:521-526.

8. Fury MG, Sherman E, Haque S, et al. A phase I study of daily everolimus plus low-dose weekly cisplatin for patients with advanced solid tumors. Cancer Chemother Pharmacol. 2012;69:591-598.

9. Novartis Pharmaceuticals Corporation. Afinitor ${ }^{\circledR}$ (everolimus) tablets for oral administration [package insert]. Available at: http://www. rxlist.com/afinitor-drug/indications-dosage.htm. Accessed October 21, 2015.

10. Neshat MS, Mellinghoff IK, Tran C, et al. Enhanced sensitivity of PTEN-deficient tumors to inhibition of FRAP/mTOR. Proc Natl Acad Sci USA. 1998;18:10314-10319.

11. O'Reilly T, McSheehy PM, Wartmann M, et al. Evaluation of the mTOR inhibitor, everolimus, in combination with cytotoxic antitumor agents using human tumor models in vitro and in vivo. Anticancer Drugs. 2011;22:58-78.

12. Wu L, Birle DC, Tannock I. Effects of the mammalian target of rapamycin inhibitor CCI-779 used alone or with chemotherapy on human prostate cancer cells and xenografts. Cancer Res. 2005;65: 2825-2831.

13. Grünwald V, Degraffenried L, Russel D, et al. Inhibitors of mTOR reverse doxorubicin resistance conferred by PTEN status in prostate cancer cells. Cancer Res. 2002;62:6141-6145.

14. Eisenhauer EA, Therasse P, Bogaerts J, et al. New response evaluation criteria in solid tumours: revised RECIST guideline (version 1.1). Eur J Cancer. 2009;45:228-247.

15. Scher H, Halabi S, Tannock I, et al. Design and end points of clinical trials for patients with progressive prostate cancer and castrate levels of testosterone: recommendations of the Prostate Clinical Trials Working Group. J Clin Oncol. 2008;26:1148-1159.

16. Lawless JF. Statistical Models and Methods for Lifetime Data Analysis. 2nd ed. New York: Wiley; 1982; 2003:149.

17. Lee ET, Wang JW. Statistical Methods for Survival Data Analysis. 3rd ed. New York: Wiley; 2003:76-91.

18. Knowles MA, Habuchi T, Kennedy W, Cuthbert-Heavens D. Mutation spectrum of the $9 \mathrm{q} 34$ tuberous sclerosis gene TSC1 in transitional cell carcinoma of the bladder. Cancer Res. 2003;63: $7652-7656$.

19. Hauke RJ, Infante JR, Rubin MS, et al. Everolimus in combination with paclitaxel and carboplatin in patients with metastatic melanoma: a phase II trial of the Sarah Cannon Research Institute Oncology Research Consortium. Melanoma Res. 2013;23:468-473.

20. Aparicio AM, Harzstark AL, Corn PG, et al. Platinum-based chemotherapy for variant castrate-resistant prostate cancer. Clin Cancer Res. 2013;19:3621-3630.

21. Iyer G, Hanrahan AJ, Milowsky MI, et al. Genome sequencing identifies a basis for everolimus sensitivity. Science. 2012;338:221.

22. Templeton AJ, Dutoit V, Cathomas R, et al. Phase 2 trial of singleagent everolimus in chemotherapy-naive patients with castrationresistant prostate cancer (SAKK 08/08). Eur Urol. 2013;64:150-158.

23. Nakabayashi M, Werner L, Courtney KD, et al. Phase II trial of RAD001 and bicalutamide for castration-resistant prostate cancer. BJU Int. 2012;110:1729-1735.

24. Xu Y, Chen SY, Ross KN, Balk SP. Androgens induce prostate cancer cell proliferation through mammalian target of rapamycin activation and post-transcriptional increases in cyclin D proteins. Cancer Res. 2006;66:7783-7792.

25. Kaarbø M, Mikkelsen OL, Malerød L, et al. PI3K-AKT-mTOR pathway is dominant over androgen receptor signaling in prostate cancer cells. Cell Oncol. 2010;32:11-27.

26. Marhold M, Tomasich E, El-Gazzar A, et al. HIF1 $\alpha$ regulates mTOR signaling and viability of prostate cancer stem cells. Mol Cancer Res. 2015;13:556-564.

\section{APPENDIX}

\section{Supplementary Data}

Supplementary data associated with this article can be found, in the online version, at http://dx.doi.org/10.1016/j.urology. 2015.08.008. 